\title{
Oxidative stability of palm - and soybean-based medium- and long-chain triacylglycerol (MLCT) oil blends
}

\begin{abstract}
BACKGROUND: Medium- and long-chain triacylglyerols (MLCT) enzymatically esterified using Lipozyme RM IM lipase has very low oxidative stability as it does not contain any antioxidants. The aim of this work was to study the ability of various antioxidants to increase the oxidative stability of palm- and soybean-based MLCT blends which assist to bring up the oxidative stability of both MLCT blends. In this study, the effectiveness of rosemary extracts, sage extracts, tert-butylhydroquinone (TBHQ) and mixtures of tert-butyl-4-hydroxyanisole (BHA) and tert-butyl-p-hydroxytoluene (BHT) in protecting against oxidation of various MLCT blends was investigated. RESULTS: Blending of MLCT oil with either palm olein or soybean oil improved its smoke point values and oxidative stability. TBHQ addition to both palm- and soybean-based MLCT blends increased oxidative stability. Combination of BHA and BHT showed no significant improvement $(\mathrm{P}>0.05)$ in ability to protect blends from oxidation compared to natural antioxidants such as sage or rosemary extracts. CONCLUSION: Blended oils with $500 \mathrm{~g} \mathrm{~kg}-1$ MLCT and $500 \mathrm{~g} \mathrm{~kg}-1$ palm olein (MP5) were the most suitable for use at high temperature based on the fatty acid composition of the MLCT blends, which subsequently had an effect on thermal oxidative stability. In general, addition of either natural or synthetic antioxidant assisted in improving the antioxidative strength of both MLCT blends. MLCT blends with added TBHQ showed the highest thermal oxidative stability among the antioxidants used. Copyright (C) 2008 Society of Chemical Industry
\end{abstract}

Keyword: Medium and long-chain triacylglycerols (MLCT); Induction time; Rosemary extracts; Sage extracts; BHA; BHT; TBHQ; smoke point. 